\title{
Dendrimer Heparan Sulfate Glycomimetics: Potent Heparanase Inhibitors for Anticancer Therapy
}

Olga V. Zubkova, ${ }^{* \dagger \odot ~ Y a s s i r ~ A . ~ A h m e d, ~}{ }^{\ddagger}$ Scott E. Guimond, ${ }^{\ddagger}$ Sophia-Louise Noble, ${ }^{\S}$ John Holmes Miller, ${ }^{\S}$ Raymond Alexander Alfred Smith, ${ }^{\circledR}{ }^{(0)}$ Victor Nurcombe, ${ }^{\&}$ (i) Peter C. Tyler, ${ }^{\dagger}{ }^{\circ}$ Marina Weissmann, ${ }^{\#}$ Israel Vlodavsky," and Jeremy E. Turnbull ${ }^{\ddagger}$

${ }^{\dagger}$ The Ferrier Research Institute, Victoria University of Wellington, Gracefield Research Centre, Lower Hutt, New Zealand

${ }^{\ddagger}$ Dept. of Biochemistry, Institute of Integrative Biology, University of Liverpool, Liverpool L69 7ZB, United Kingdom

${ }^{\S}$ School of Biological Sciences, Victoria University of Wellington, Kelburn, Wellington, New Zealand

${ }^{\&}$ Glycotherapeutics Group (VNSC), Institute of Medical Biology, Agency for Science, Technology and Research (A*STAR), 138632 Singapore

${ }^{\#}$ Cancer and Vascular Biology Research Center, Rappaport Faculty of Medicine, Technion-Israel Institute of Technology, Haifa, Israel

\section{Supporting Information}

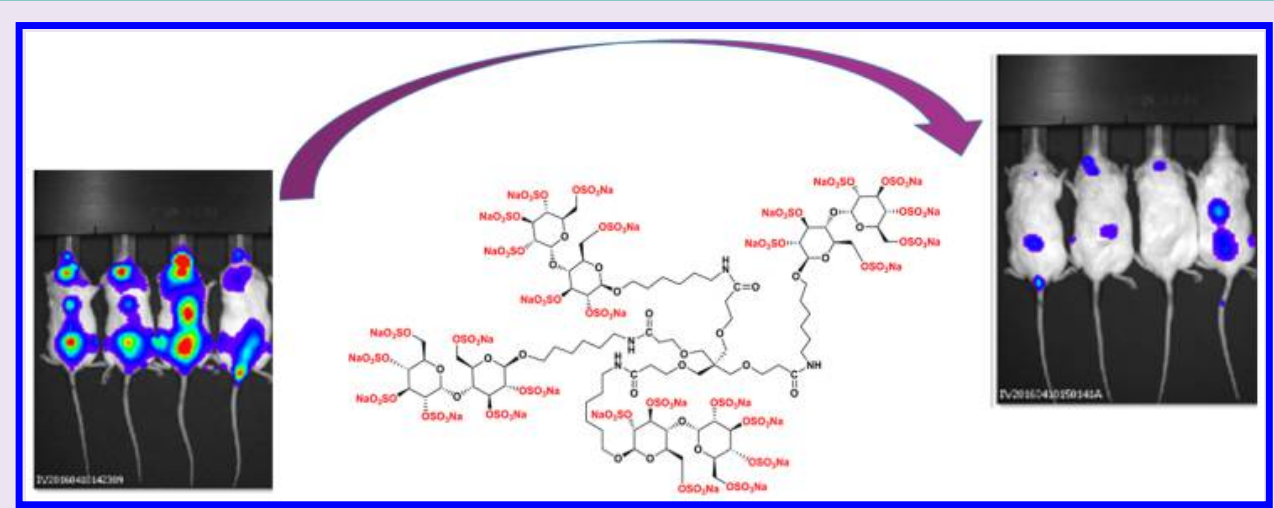

ABSTRACT: Heparanase is a mammalian endoglycosidase that cleaves heparan sulfate (HS) polysaccharides and contributes to remodelling of the extracellular matrix and regulation of HS-binding protein bioavailabilities. Heparanase is upregulated in malignant cancers and inflammation, aiding cell migration and the release of signaling molecules. It is established as a highly druggable extracellular target for anticancer therapy, but current compounds have limitations, because of cost, production complexity, or off-target effects. Here, we report the synthesis of a novel, targeted library of single-entity glycomimetic clusters capped with simple sulfated saccharides. Several dendrimer HS glycomimetics display low nM $\mathrm{IC}_{50}$ potency for heparanase inhibition equivalent to comparator compounds in clinical development, and potently inhibit metastasis and growth of human myeloma tumor cells in a mouse xenograft model. Importantly, they lack anticoagulant activity and cytotoxicity, and also inhibit angiogenesis. They provide a new candidate class for anticancer and wider therapeutic applications, which could benefit from targeted heparanase inhibition.

T eparan sulfate (HS) is a highly sulfated glycosaminoglycan with a variety of critical functions in cell signaling and regulation. ${ }^{1} \mathrm{HS}$ acts as an extracellular storage depot of growth factors, angiogenic proteins, and chemokines, and also serves to regulate ligand-receptor interactions at the cell surface. $^{2,3}$ Heparanase is the only known endo- $\beta$-D-glucuronidase capable of cleaving HS side chains of heparan sulfate proteoglycans (HSPGs) into shorter, active fragments. ${ }^{4,5}$ Cleavage of HS by heparanase facilitates structural alterations of the extracellular matrix (ECM) and contributes to releasing various sequestered HS-binding proteins. ${ }^{6}$ Heparanase is upregulated in malignant cancers, and its overexpression correlates with angiogenesis, increased tumor size, enhanced metastasis, and poor prognosis. ${ }^{7,8}$ The upregulation of heparanase in malignant cancer also correlates closely with increases in new blood vessels around tumors, providing a ready pathway for cancer cell entry into the circulation. ${ }^{9}$ This makes heparanase a promising extracellular target for new antitumor treatments.

While HS oligosaccharides can mimic or interfere with biological systems, their exploitation has been hindered by the complexity of their synthesis. Recently, we developed a novel

Received: October 10, 2018

Accepted: November 27, 2018

Published: November 27, 2018 
Scheme 1. Synthesis of Glycomimetics: (A, B) Synthetic Targets, (C) Preparation of Saccharide Fragments, ${ }^{a}$ (D) Preparation of Tetramer Dendritic Cores, ${ }^{b}$ and (E) Preparation of HS Glycomimetics ${ }^{c}$

A.

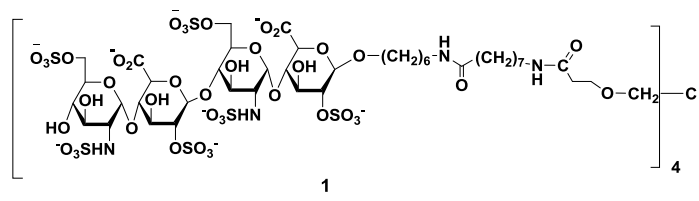

C.

E.

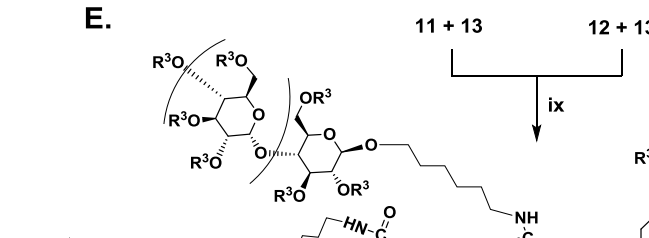

B.

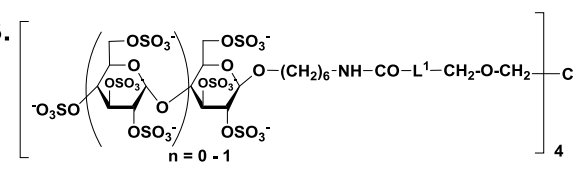

$\mathrm{L}^{1}=\mathrm{a}, \mathrm{CH}_{2} ; \mathrm{b}, \mathrm{CH}_{2} \mathrm{CONH}\left(\mathrm{CH}_{2}\right)_{7} ; \mathrm{c}, \mathrm{CH}_{2} \mathrm{CO}-\mathrm{NH}\left(\mathrm{CH}_{2}\right)_{7}-\mathrm{CONH}-\left(\left(\mathrm{CH}_{2}\right)_{2} \mathrm{O}\right)_{3}-\left(\mathrm{CH}_{2}\right)_{2}$ $26-31$

D.
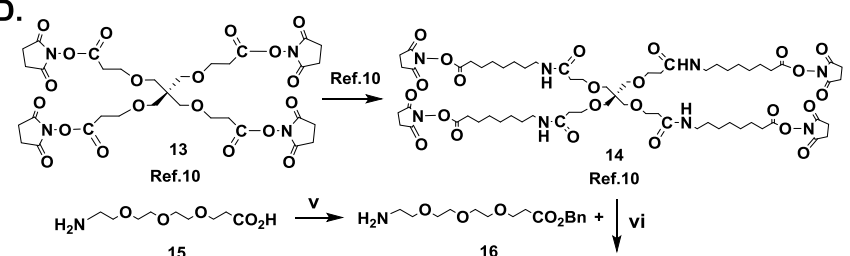

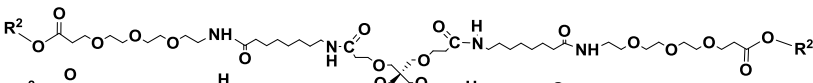

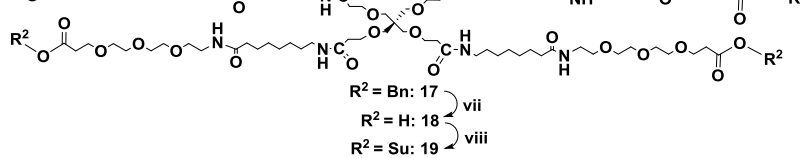

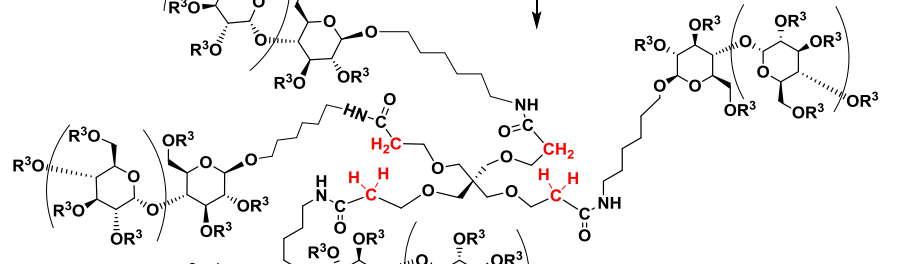

$11+14 \quad 12+14$

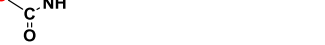

$x\left(R^{3}=H: 20: n=0, L^{1}=C_{2} ; 23: n=1, L^{1}=C_{2}\right.$

$\mathrm{R}^{3}=\mathrm{SO}_{3}: 26: n=0, \mathrm{~L}^{1}=\mathrm{CH}_{2} ; 29: n=1, \mathrm{~L}^{1}=\mathrm{CH}_{2}$

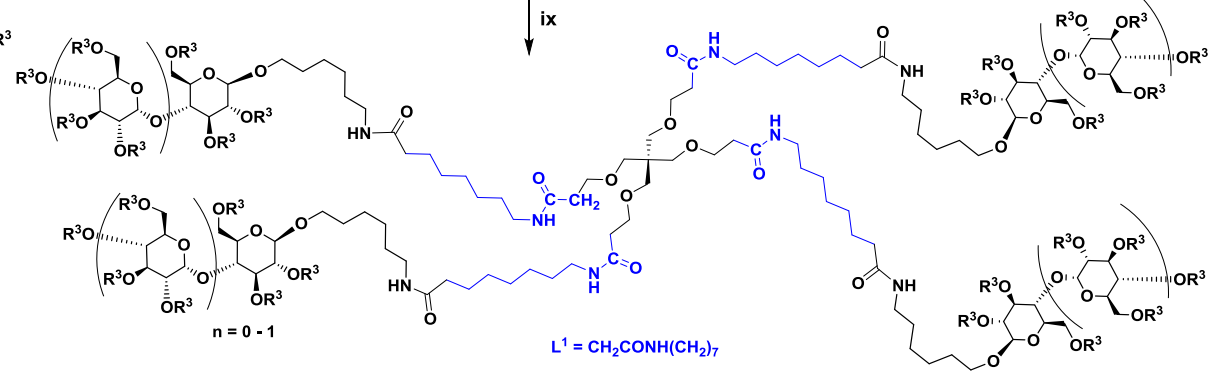

$x\left(\begin{array}{l}R^{3}=\mathrm{H}: 21: n=0, \mathrm{~L}^{1}=\mathrm{CH}_{2} \mathrm{CONH}\left(\mathrm{CH}_{2}\right)_{7} ; 24: n=1, \mathrm{~L}^{1}=\mathrm{CH}_{2} \mathrm{CONH}\left(\mathrm{CH}_{2}\right)_{7} \\ \mathrm{R}^{3}=\mathrm{SO}_{3}^{-}: 27: n=0, \mathrm{~L}^{1}=\mathrm{CH}_{2} \mathrm{CONH}\left(\mathrm{CH}_{2}\right)_{7} ; 30: n=1, \mathrm{~L}^{1}=\mathrm{CH}_{2} \mathrm{CONH}\left(\mathrm{CH}_{2}\right)_{7}\end{array}\right.$

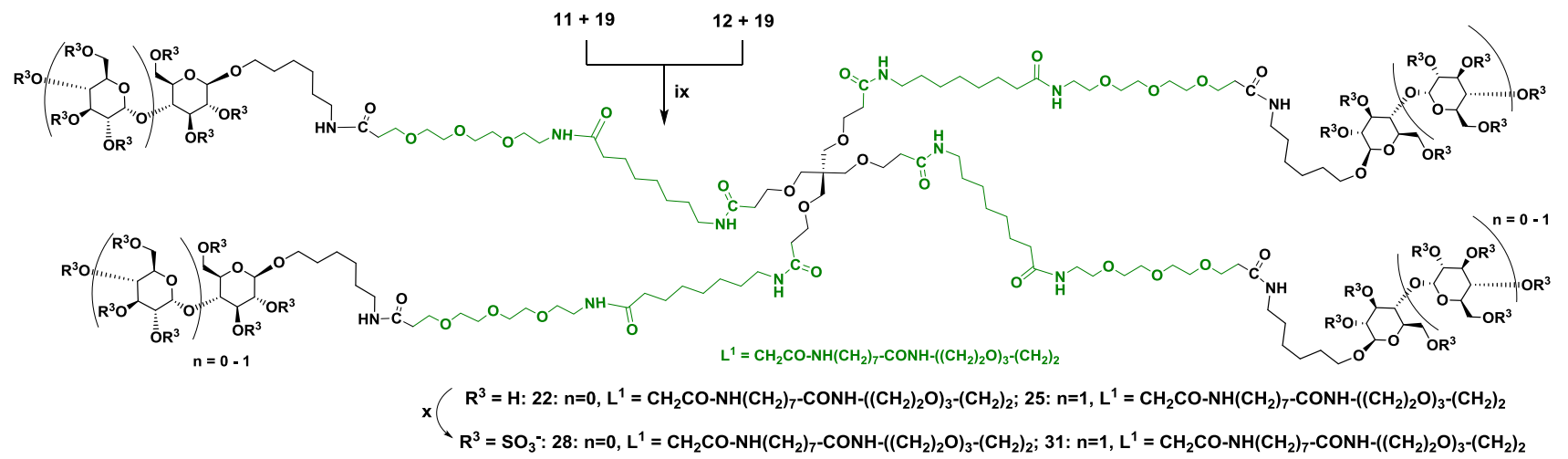

${ }^{a}(\mathrm{i}) \mathrm{BF}_{3} \cdot \mathrm{OEt}_{2}, \mathrm{DCM}, \mathrm{RT}$; (ii) $\mathrm{NaN}_{3}, \mathrm{DMF}, 80^{\circ} \mathrm{C}$; (iii) $\mathrm{NaOMe}, \mathrm{MeOH}, \mathrm{RT}$; and (iv) $\mathrm{Ra}-\mathrm{Ni}_{1} \mathrm{H}_{2}$, aqueous $\mathrm{MeOH}, \mathrm{RT} .{ }^{b}(\mathrm{v}) \mathrm{BnOH}, \mathrm{SOCl}{ }_{2} 0$ to $100{ }^{\circ} \mathrm{C}$; (vi) $\mathrm{Et}_{3} \mathrm{~N}, \mathrm{DMF}, \mathrm{RT}$; (vii) $\mathrm{H}_{2}, \mathrm{Pd}(\mathrm{OH})_{2} / \mathrm{C}$, aq. $\mathrm{MeOH}, \mathrm{RT}$; and (viii) NHS, EDC, DMF, DIPEA, RT. ${ }^{c}(\mathrm{ix}) \mathrm{Et}_{3} \mathrm{~N}, \mathrm{DMF}, \mathrm{RT}$; and $(\mathrm{x})$ $\mathrm{SO}_{3} \cdot \mathrm{NMe}_{3}, \mathrm{DMF}, 60^{\circ} \mathrm{C}$.

synthetic approach to more accessible polyvalent HS-mimetic constructs with potential advantages as therapeutics. ${ }^{10} \mathrm{We}$ employed the clustering effect of dendrimers in which weak interactions of small sugar fragments are significantly enhanced by appending multiple copies of specific sugars on defined chemical scaffolds. ${ }^{11}$ Our targeted libraries of single-entity HS oligosaccharides and glycomimetic clusters have already been demonstrated to mimic longer natural HS molecules that have 

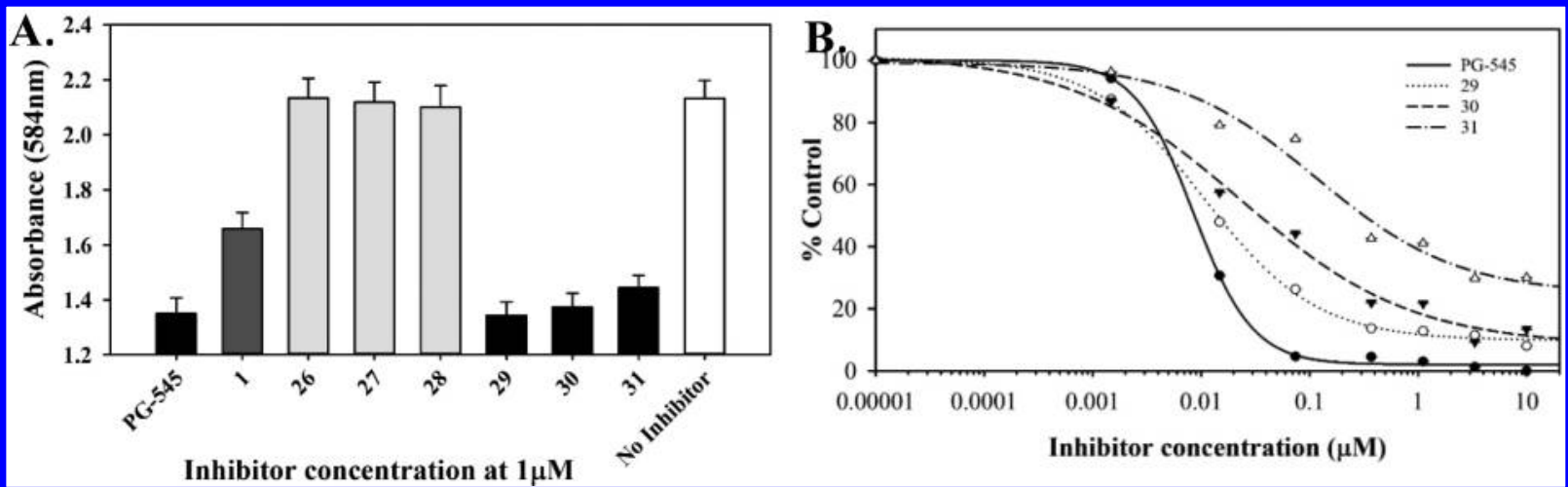

Figure 1. Heparanase inhibitory activity of selected compounds. (A) Initial in vitro screen of heparanase inhibitory activity of selected compounds at a $1 \mu \mathrm{M}$ concentration in a Fondiparinux substrate assay using $100 \mathrm{nM}$ heparanase. (B) Dose response curves for heparanase inhibition by selected compounds in the Fondiparinux assay using $10 \mathrm{nM}$ heparanase. $\mathrm{IC}_{50}$ values were calculated from the curves (see the Methods section in the Supporting Information: PG545, $8 \mathrm{nM}$; 29, $11 \mathrm{nM}$; 30, $23 \mathrm{nM}$; 31, $116 \mathrm{nM}$ ).

a role in Alzheimer's disease. ${ }^{12-14}$ The ability of the target compounds to inhibit the BACE-1 protease was investigated using fluorescence resonance energy transfer (FRET) peptide cleavage assays. All of the glycomimetic clusters inhibited BACE-1 with half maximal inhibitory concentration $\left(\mathrm{IC}_{50}\right)$ values in the micromolar to low nanomolar range and showed a lack of off-target anticoagulant activity.

We reasoned that our dendritic cores and associated methodology would provide a novel template for the polyvalent presentation of other HS-mimicking saccharides for other indications. Here, we extended our research into anticancer applications and screened a library of HSglycomimetics for their ability to inhibit heparanase.

Our approach was to alter the ECM of tumor cells and their detachment prior to metastasis by preventing heparanase action and thereby altering the oxygen, nutrient, and growth factor supply to the tumor. Standard chemotherapeutic anticancer drugs kill rapidly dividing cancer cells but also affect normal cell proliferation, such as that which occurs in the bone marrow and gut, leading to toxic side effects. In contrast, normal cells express extracellular heparanase at very low levels, and heparanase knockout animals exhibit no obvious deficits. These characteristics imply that inhibition of heparanase will cause minimal side effects in cancer patients.

Initially, we tested the available $N$-acetylated $\mathrm{HS}$ mimetics developed for BACE1 inhibition; ${ }^{10}$ but these did not inhibit heparanase with any significant potency. However, one of the $\mathrm{N}$-sulfated analogues (compound 1, Scheme 1A) did show modest inhibitory activity $(60 \%$ inhibition at a concentration of $1 \mu \mathrm{M}$; see Figure 1A). Thus, we developed further analogues (26-31; see Scheme 1B), reasoning that increasing the level of sulfation would lead to more potent inhibitors. As a simplified alternative to complex HS saccharides, we synthesized a novel targeted library of single-entity dendrimer HS glycomimetic clusters capped with simple sulfated saccharides, glucose, and maltose, that potentially mimic gluco-configured HS. We also varied the length of the dendritic core arms employing PEG linkers to increase the solubility of the glycomimetics. Using affordable starting ingredients, we also significantly shortened the synthesis process, thus markedly reducing manufacturing costs.

Glycosylation of peracetylated sugars, ${ }^{15}$ glucose (2), and maltose (3) with 6-chloroethanol (4) in dichloromethane in the presence of boron trifluoride etherate at RT gave glycosides (5) and (6). Displacement of the chloro-group with sodium azide in DMF at $80{ }^{\circ} \mathrm{C}$ followed by Zemplen deacetylation afforded azido-derivatives (9) and (10). Reduction of the azides at ambient temperature and pressure with Raney Nickel under hydrogen in aqueous methanol furnished amino-glycosides (11) and (12) in quantitative yields (Scheme 1C). "Short-armed" tetra- $N$-hydroxysuccinimide activated ester 13 and a "long-armed" tetra- $N$ hydroxysuccinimide activated ester 14 were prepared as described in our earlier work. ${ }^{10}$ Treatment of PEG-acid (15) with benzyl alcohol and thionyl chloride gave a benzyl ester of PEG amino acid 16. Coupling the benzyl ester 16 to a "longarmed" core 14 led to the tetravalent dendritic core 17 . Hydrogenolysis of (17) gave the tetra-acid 18, and its treatment with NHS/EDC afforded the PEGylated $\mathrm{N}$ hydroxysuccinimide ester 19 (Scheme 1D).

In our previous work, we observed complex mixtures of starting material, di-, tri-, and tetra-substituted clusters, when sulfated HS fragments were attached to the tetrameric dendritic cores. This was likely due to the electrostatic repulsion of anionic sulfo-groups. We improved the coupling yields by first attaching non-charged fragments followed by oversulfation. Here, we followed the same strategy (Scheme 1E).

Coupling of four equivalents of glycoside fragments 11 and 12 with tetra- $N$-hydroxysuccinimide active esters 13,14 , and

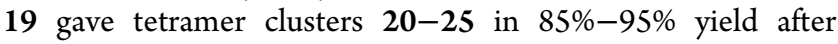
chromatography. Sulfation of tetramers in dry DMF under argon at $60{ }^{\circ} \mathrm{C}$ for $72 \mathrm{~h}$ afforded the desired $\mathrm{O}$-sulfated HS mimetics $26-31$ in excellent yields of $88 \%-92 \%$ after chromatography.

All six final products $26-31$ and their six precursors $20-25$ were analyzed by one-dimensional (1D) and two-dimensional (2D) NMR spectroscopy in deuterium oxide at $500 \mathrm{MHz}$. The chemical shift data are presented in the Supporting Information (Tables S1 and S2). Chemical shifts were assigned using the HSQC technique. ${ }^{1} \mathrm{H}$ and ${ }^{13} \mathrm{C}$ NMR spectra indicated that compounds 26-31 were fully sulfated, since single sets of peaks were observed with resonances shifted downfield.

The ability of the target compounds to inhibit heparanase in vitro was investigated using a Fondaparinux substrate heparanase assay ${ }^{16}$ (see the Methods section in the Supporting Information), and demonstrated that HS glycomimetic clusters 


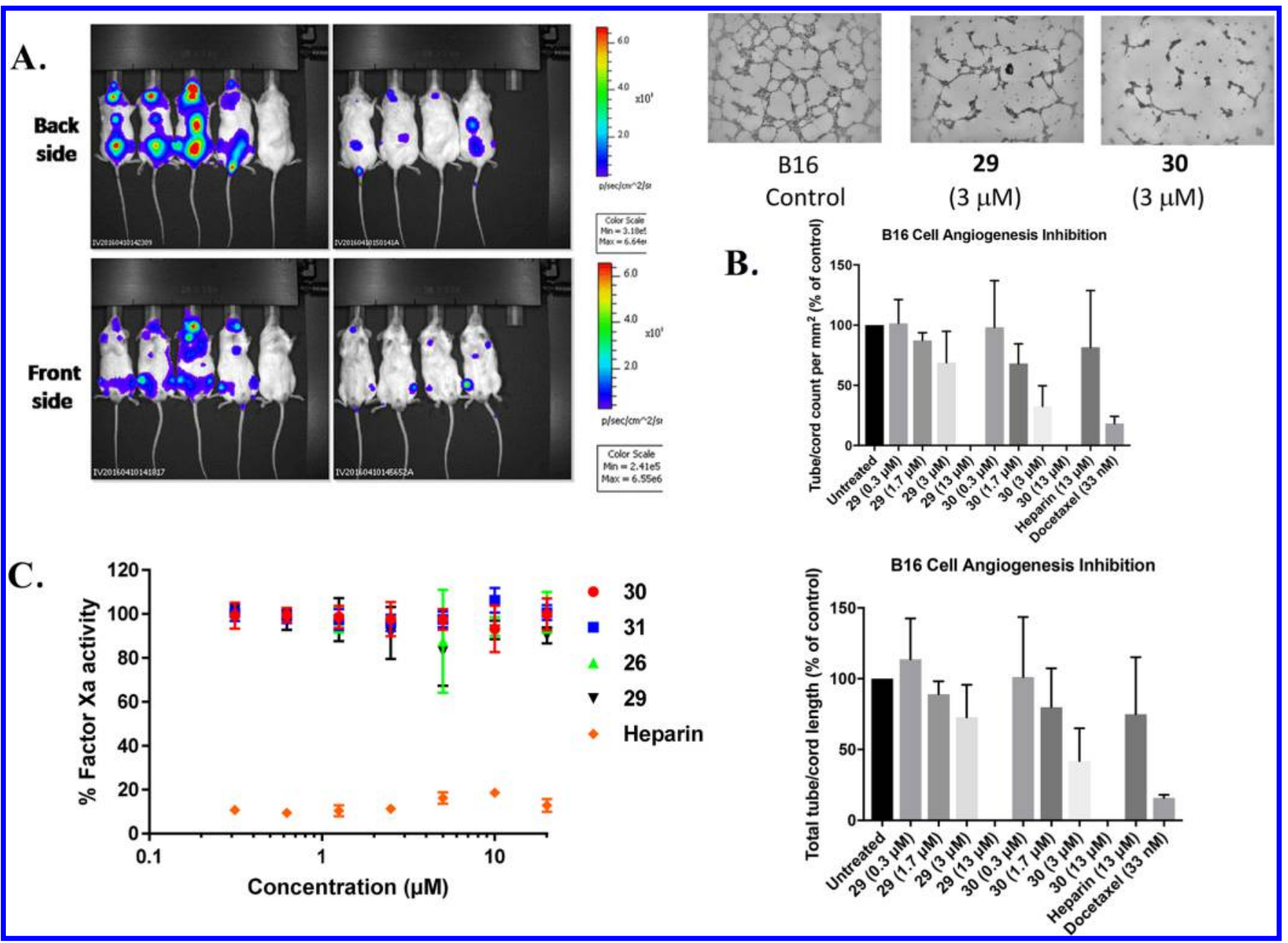

Figure 2. Screen of bioactivities of selected compounds. (A) Inhibition of metastasis and tumor growth in an immunodeficient mouse xenograft model with human myeloma cells. (See the Methods section and Figures S1 and S2 for experimental details.) On the left are untreated mice with malignant myeloma tumors containing a luciferase reporter, 4 weeks after injection of tumor cells. A control mouse that was not injected with cells is included (No. 5 from the left). On the right are mice injected with myeloma cells, and treated for 4 weeks with our heparanase inhibitor 30 (600 $\mu \mathrm{g}$ per day). (B) Inhibition of angiogenesis. The murine melanoma cell line B16 is capable of vascular mimicry, a process similar to angiogenesis. The effect of the dendrimer HS glycomimetics 29 and $\mathbf{3 0}$ were tested to see if they could prevent vascular mimicry in B16 cultures grown in a basement membrane extract (Cultrex BME) (see the Methods section). Images of epithelial tubes or cords are shown in the upper part of Panel B, and a summary of the results after treatment for $18 \mathrm{~h}$ with $\mathbf{2 9}$ or $\mathbf{3 0}$ is shown on the lower part, presented as the total number of tubes/cords and the total length of tubes/cords $(n=3$ experiments; mean \pm SEM). Heparin was used as a negative control, and docetaxel (DTX) was used as a positive control for inhibition of angiogenesis. (C) Inhibition of Factor Xa anticoagulant activity by HS glycomimetics $(n=6$ replicates; mean \pm $\mathrm{SD})$.

(26-31) inhibited heparanase with $\mathrm{IC}_{50}$ values in the micromolar to low nanomolar range (Figure 1).

Tetramers 26-28 with sulfated monosaccharide (glucose) fragments showed only weak ability to inhibit heparanase in an initial screen at $1 \mu \mathrm{M}$ dosage (Figure 1 , Panel A), with $\mathrm{IC}_{50}$ values of $\gg 1 \mu \mathrm{M}$; in contrast, tetramers 29-31 with larger sulfated disaccharide (maltose) residues were the most active variants (Figure 1A). Therefore, a quantity of at least two sulfated monosaccharide sugar units was critical for activity. Dose response experiments indicated that the potency was higher for "long-armed" PET tetramer 30 (23 nM), compared to the "PEGylated long-armed" cluster with sulfated maltose, 31 (116 nM; Figure 1B). Interestingly, a "short-armed" tetramer cluster 29 with sulfated maltose fragments showed the highest potency, with an $\mathrm{IC}_{50}$ value of $11 \mathrm{nM}$. These results indicate that the length of the arms is a significant factor in determining potency; this, along with potential activity of dimer constructs, will be a useful avenue to explore in further optimization studies. The potency of the known heparanase inhibitor, PG545, was found to be $8 \mathrm{nM}$ (Figure 1B), in very good agreement with the literature. ${ }^{28}$ Thus, 29 and 30 have potency very similar to the comparator PG545, which is in clinical development. ${ }^{25}$

Myeloma tumors express high levels of the HSPGs on their cell surfaces. ${ }^{17}$ Heparanase is upregulated in myeloma tumor cells, and the bone marrow plasma of some patients provides a rich source of biologically active fragments that enhance a tumor's growth and metastasis. ${ }^{18}$ Previous work has shown that heparanase inhibitors reduce myeloma metastasis and tumor growth in a xenograft mouse model. ${ }^{17,19}$ We tested one of our most potent in vitro inhibitors 30 in this highly aggressive myeloma model (Figure 2A), in which cells metastasize to bone marrow with tumors growing predominantly in the bone, closely mimicking these aspects of the human disease. ${ }^{17}$ We observed remarkably reduced tumor spread and growth in the mice treated with the inhibitor 

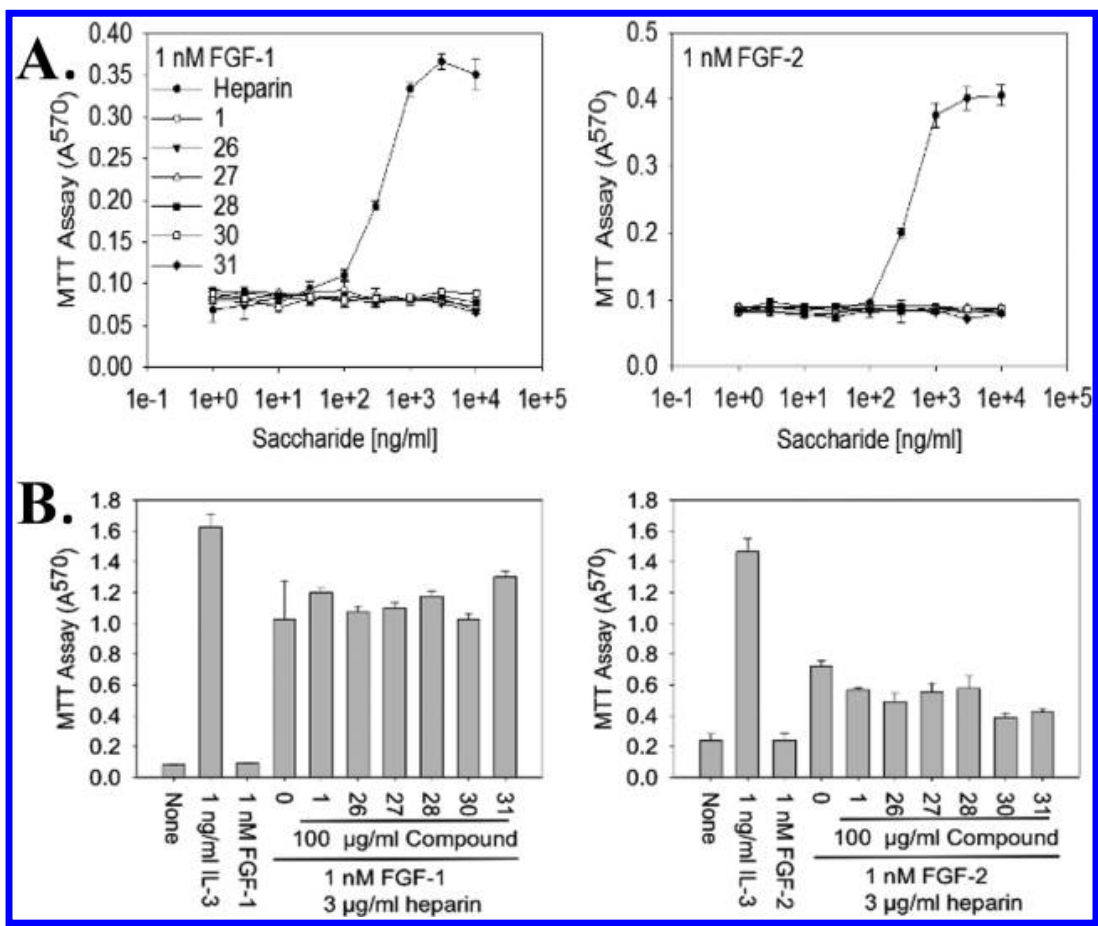

Figure 3. Screen of FGF signaling regulation by selected compounds. (A) No HS glycomimetic activation of proliferation of BaF3 cells transfected with FGFR1c by FGF-1 or FGF-2. Left graph shows the proliferation activity of $1 \mathrm{nM} \mathrm{FGF-1}$ in the presence of increasing concentrations of heparin or compounds; the right graph is the same as left graph but with $1 \mathrm{nM} \mathrm{FGF-2.} \mathrm{See} \mathrm{the} \mathrm{Methods} \mathrm{section} \mathrm{for} \mathrm{experimental} \mathrm{details.} \mathrm{(B)}$ Inhibition of heparin-induced activation of FGFR1c. FGF2 action is inhibited but not FGF1 by selected compounds. In the graph on the left, 100 $\mu \mathrm{g} / \mathrm{mL}$ of compounds were added to BaF3 cells transfected with FGFR1c in the presence of $1 \mathrm{nM} \mathrm{FGF}-1$ and $3 \mu \mathrm{g} / \mathrm{mL}$ heparin. Cells exposed to no growth factor, $1 \mathrm{ng} / \mathrm{mL}$ IL-3 or FGF-1 alone are also shown. The graph on the right is the same as the left graph, but with $1 \mathrm{nM}$ FGF-2. (See the Methods section for experimental details.)

(dorsal view, $85.4 \%$ inhibition, $p=0.037$ and ventral view, $88.5 \%$ inhibition, $p=0.024$ at 4 weeks; see the Methods section and Figure S2 in the Supporting Information), indicative of potent heparanase inhibition.

Some cancer cells form pre-capillary-like tubes or cords when grown in a basement membrane gel extract, using a process called vascular mimicry. ${ }^{20}$ Both glycomimetics 29 and 30 inhibited vascular mimicry in cultures of B16 melanoma cells at concentrations of 10 and $40 \mu \mathrm{M}$ (Figure 2B), without inhibiting cell migration (Figure $\mathrm{S} 3$ in the Supporting Information). Vascular mimicry, which is a process similar to angiogenesis, generates blood vessels for vascularization of tumors and occurs in the absence of an endothelial cell line in the cultures. Prevention of neovascularization of a tumor would contribute to inhibition of a tumor's growth by the HS glycomimetics.

A notable potential side effect of heparin and related compounds that can limit their wider clinical application is anticoagulant activity. Importantly, the compounds that we synthesized displayed a remarkable lack of any significant anticoagulant activity toward Factor $\mathrm{Xa}$ at concentrations $>100$-fold or higher than their $\mathrm{IC}_{50}$ for inhibiting heparanase (Figure 2C), indicating that they differ from some other compounds in this class, such as PG545 $5^{23}$ and necuparanib. ${ }^{29}$ Importantly, we also established that this class of compounds was not cytotoxic to BaF3 mouse lymphoid cells at $100 \mu \mathrm{g} / \mathrm{mL}$ $(\sim 15-32 \mu \mathrm{M})$, which is $\sim 100-1500$ fold higher than their $\mathrm{IC}_{50}$ for inhibiting heparanase (see Figure S4 in the Supporting Information).

A further known activity of heparin is regulation of fibroblast growth factors (FGFs), which are crucial for cell growth and can also promote angiogenesis. ${ }^{21}$ We observed no activation of FGF-1 or FGF-2 signaling via FGF receptor FGFR1c with any of the compounds on BaF3 lymphoid cell proliferation (26-31 Figure $3 \mathrm{~A}$ ), where viability is dependent on FGF signaling in the absence of Interleukin 3 (IL-3) (see the Methods section).

We also examined the compounds for inhibitory properties toward FGF signaling in $\mathrm{BaF} 3$ cells in which cells are partially activated by the addition of $3 \mu \mathrm{g} / \mathrm{mL}$ heparin. Notably, none of the compounds (1, 26-31; see Figure 3B) significantly inhibited FGF1 in BaF3 cells transfected with FGFR1c. In contrast, FGF2 was inhibited by some of the compounds, most potently by compounds 30 and $\mathbf{3 1}$. This is significant since inhibition of FGF2 could contribute to the antiangiogenic properties of these compounds that we observed (Figure 2B), providing an additional anticancer mode of action. BaF3 cells are a mouse pre-B lymphocytic cell line dependent on IL-3 for survival. $^{22}$ They are devoid of heparan sulfate and FGF receptors and do not respond to FGF in the presence or absence of heparin. ${ }^{23} \mathrm{BaF} 3$ cells transfected with FGFR1c gain the ability to respond to FGF-1 and FGF-2 in the presence of exogenously added heparin or heparin mimetics, and the cells can proliferate normally in the absence of $\mathrm{Il}-3^{23}$ (Figure $3 \mathrm{~A}$ ). If no heparin is present or a heparin species is not capable of supporting FGF activity, the cells do not proliferate and eventually die.

There are no therapeutically effective heparanase inhibitors currently available for use in the clinic. ${ }^{24}$ Heparin, which is an antithrombotic drug that also inhibits heparanase, is not clinically useful, because of its strong anticoagulant activity. However, the use of HS-related compounds as antitumor agents has been reported in the literature, resulting in clinical 
trials that are currently underway. For example, Progen Pharmaceuticals has designed a compound called PG545 that is produced from a very expensive tetrasaccharide maltotetraose. $^{25}$ PG545 treatment reduces heparanase activity in primary tumors and is presently undergoing clinical trials. ${ }^{26}$

The heparanase inhibitors necuparanib (Momenta Pharmaceuticals) $)^{27}$ and a glycol-split modified heparin roneparstat (Leadiant Biosciences) ${ }^{28}$ are produced from natural heparin by chemical modifications. These compounds display multiple anticancer activities, including heparanase inhibition and antiangiogenic properties. However, because they are complex mixtures, they may face hurdles in obtaining FDA approval, as the methods required to characterize batch-to-batch variations are very elaborate and costly. Furthermore, both PG545 ${ }^{23}$ and necuparanib ${ }^{29}$ also retain significant anticoagulant activity, and roneparstat requires high dosage levels, because of the short half-life; ${ }^{27}$ factors that might limit their clinical usage.

Thus, collective data point to heparanase as a good target molecule to interfere with cancer progression, though none of the treatments already under development are ideal. Our HS glycomimetics could provide a solution to these drawbacks, since they have distinct advantages, including a unique combination of potency comparable to PG545 (currently the most potent of the described inhibitors, with an $\mathrm{IC}_{50}$ value of $12 \mathrm{nM}),{ }^{30}$ allied with a lack of any significant anticoagulant activity, and also lack of cell toxicity. We have described rapid, convenient, and economical methods for the synthesis of a range of single chemical entity sulfated glycomimetics. Further studies are underway to evaluate their pharmacokinetics, bioavailability, and safety as a prelude to potential clinical testing.

In summary, we have, for the first time, prepared chemically defined, single-entity polyvalent heparanase inhibitors displaying simple sulfated sugars on dendritic cores. We have shown that these clusters inhibit heparanase with potency equivalent to the comparator PG545, as well as inhibiting angiogenesis and FGF2, and they also completely lack off-target anticoagulant activity and cell toxicity. The compounds reported here are a novel class of anticancer candidates with additional potential applications in diseases where targeting of heparanase inhibition is expected to be beneficial, including inflammatory disorders, ${ }^{31}$ diabetic nephropathy, ${ }^{32}$ fibrosis, ${ }^{33}$ and viral pathogenesis. ${ }^{34,35}$

\section{METHODS}

All methods are described in the Supporting Information.

\section{ASSOCIATED CONTENT}

\section{S Supporting Information}

The Supporting Information is available free of charge on the ACS Publications website at DOI: 10.1021/acschembio.8b00909.

Materials, methods, abbreviations, supplementary figures, detailed description of the synthetic procedures and NMR spectra (PDF)

\section{AUTHOR INFORMATION}

\section{Corresponding Author}

*E-mail: Olga.Zubkova@vuw.ac.nz.

ORCID

Olga V. Zubkova: 0000-0002-6892-8812

John Holmes Miller: 0000-0001-6383-1037
Raymond Alexander Alfred Smith: 0000-0002-0688-2193

Victor Nurcombe: 0000-0003-1270-7452

Peter C. Tyler: 0000-0002-3151-6208

\section{Notes}

The authors declare no competing financial interest.

\section{ACKNOWLEDGMENTS}

The authors thank H. Wong and Y. Lu for an excellent NMR and mass spectrometry service, and V. Ferro for the gift of PG545, prepared according to the literature. ${ }^{23}$

\section{REFERENCES}

(1) Xu, D., and Esko, J. D. (2014) Demystifying Heparan SulfateProtein Interactions. Annu. Rev. Biochem. 83, 129-157.

(2) Bishop, J. R., Schuksz, M., and Esko, J. D. (2007) Heparan sulphate proteoglycans fine-tune mammalian physiology. Nature 446, 1030-1037.

(3) Poon, S., Lu, X., Smith, R. A. A., Ho, P., Bhakoo, K., Nurcombe, V., and Cool, S. M. (2018) Improved recovery from limb ischaemia by delivery of an affinity-isolated heparan sulphate. Angiogenesis 21, $777-791$.

(4) Sanderson, R. D., Elkin, M., Rapraeger, A. C., Ilan, N., and Vlodavsky, I. (2017) Heparanase regulation of cancer, autophagy and inflammation: New mechanisms and targets for therapy. FEBS I. 284, $42-55$.

(5) Rivara, S., Milazzo, F. M., and Giannini, G. (2016) Heparanase: a rainbow pharmacological target associated to multiple pathologies including rare diseases. Future Med. Chem. 8, 647-680.

(6) Peterson, S. B., and Liu, J. (2013) Multi-faceted substrate specificity of heparanase. Matrix Biol. 32, 223-227.

(7) Arvatz, G., Weissmann, M., Ilan, N., and Vlodavsky, I. (2016) Heparanase and cancer progression: New directions, new promises. Hum. Vaccines Immunother. 12, 2253-2256.

(8) Jia, L., and Ma, S. (2016) Recent advances in the discovery of heparanase inhibitors as anti-cancer agents. Eur. I. Med. Chem. 121, 209-220.

(9) Weissmann, M., Arvatz, G., Horowitz, N., Feld, S., Naroditsky, I., Zhang, Y., Ng, M., Hammond, E., Nevo, E., Vlodavsky, I., and Ilan, N. (2016) Heparanase-neutralizing antibodies attenuate lymphoma tumor growth and metastasis. Proc. Natl. Acad. Sci. U. S. A. 113, 704-709.

(10) Tyler, P. C., Guimond, S. E., Turnbull, J. E., and Zubkova, O. V. (2015) Single-Entity Heparan Sulfate Glycomimetic Clusters for Therapeutic Applications. Angew. Chem. Int. Ed. 54, 2718-2723.

(11) Lundquist, J. J., and Toone, E. J. (2002) The Cluster Glycoside Effect. Chem. Rev. 102, 555-578.

(12) Scholefield, Z., Yates, E. A., Wayne, G., Amour, A., McDowell, W., and Turnbull, J. E. (2003) Heparan sulfate regulates amyloid precursor protein processing by BACE1, the Alzheimer's $\beta$-secretase. I. Cell Biol. 163, 97-107.

(13) Patey, S. J., Edwards, E. A., Yates, E. A., and Turnbull, J. E. (2006) Heparin Derivatives as Inhibitors of BACE-1, the Alzheimer's $\beta$-Secretase, with Reduced Activity against Factor Xa and Other

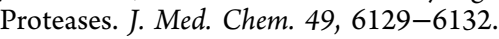

(14) Schwörer, R., Zubkova, O. V., Turnbull, J. E., and Tyler, P. C. (2013) Synthesis of a Targeted Library of Heparan Sulfate Hexa- to Dodecasaccharides as Inhibitors of $\beta$-Secretase: Potential Therapeutics for Alzheimer's Disease. Chem. - Eur. I. 19, 6817-6823.

(15) Khamsi, J., Ashmus, R. A., Schocker, N. S., and Michael, K. (2012) A high-yielding synthesis of allylglycosides from peracetylated glycosyl donors. Carbohvdr. Res. 357, 147-150.

(16) Hammond, E., Li, C. P., and Ferro, V. (2010) Development of a colorimetric assay for heparanase activity suitable for kinetic analysis and inhibitor screening. Anal. Biochem. 396, 112-116.

(17) Li, J., Pan, Q., Rowan, P. D., Trotter, T. N., Peker, D., Regal, K. M., Javed, A., Suva, L. J., and Yang, Y. (2016) Heparanase promotes 
myeloma progression by inducing mesenchymal features and motility of myeloma cells. Oncotarget 7, 11299-11309.

(18) Ramani, V. C., Zhan, F., He, J., Barbieri, P., Noseda, A., Tricot, G., and Sanderson, R. D. (2016) Targeting heparanase overcomes chemoresistance and diminishes relapse in myeloma. Oncotarget 7, 1598-1607.

(19) Ritchie, J. P., Ramani, V. C., Ren, Y., Naggi, A., Torri, G., Casu, B., Penco, S., Pisano, C., Carminati, P., Tortoreto, M., Zunino, F., Vlodavsky, I., Sanderson, R. D., and Yang, Y. (2011) SST0001, a chemically modified heparin, inhibits myeloma growth and angiogenesis via disruption of the heparanase/syndecan-1 axis. Clin. Cancer Res. 17, 1382-1393.

(20) Racordon, D., Valdivia, A., Mingo, G., Erices, R., Aravena, R., Santoro, F., Bravo, M. L., Ramirez, C., Gonzalez, P., Sandoval, A., González, A., Retamal, C., Kogan, M. J., Kato, S., Cuello, M. A., Osorio, G., Nualart, F., Alvares, P., Gago-Arias, A., Fabri, D., Espinoza, I., Sanchez, B., Corvalán, A. H., Pinto, M. P., and Owen, G. I. (2017) Structural and functional identification of vasculogenic mimicry in vitro. Sci. Rep. 7, 6985.

(21) Berry, D., Shriver, Z., Venkataraman, G., and Sasisekharan, R. (2004) Quantitative assessment of FGF regulation by cell surface heparan sulfates. Biochem. Biophys. Res. Commun. 314, 994-1000.

(22) Mathey-Prevot, B., Nabel, G., Palacios, R., and Baltimore, D. (1986) Abelson virus abrogation of interleukin-3 dependence in a lymphoid cell line. Mol. Cell. Biol. 6, 4133-4135.

(23) Ornitz, D. M., Yayon, A., Flanagan, J. G., Svahn, C. M., Levi, E., and Leder, P. (1992) Heparin is required for cell-free binding of basic fibroblast growth factor to a soluble receptor and for mitogenesis in whole cells. Mol. Cell. Biol. 12, 240-247.

(24) Ferro, V., Liu, L., Johnstone, K. D., Wimmer, N., Karoli, T., Handley, P., Rowley, J., Dredge, K., Li, C. P., Hammond, E., Davis, K., Sarimaa, L., Harenberg, J., and Bytheway, I. (2012) Discovery of PG545: A Highly Potent and Simultaneous Inhibitor of Angiogenesis, Tumor Growth, and Metastasis. I. Med. Chem. 55, 3804-3813.

(25) Dredge, K., Hammond, E., Davis, K., Li, C. P., Liu, L., Johnstone, K., Handley, P., Wimmer, N., Gonda, T. J., Gautam, A., Ferro, V., and Bytheway, I. (2010) The PG500 series: novel heparan sulfate mimetics as potent angiogenesis and heparanase inhibitors for cancer therapy. Invest. New Drugs 28, 276-283.

(26) Dredge, K., Brennan, T., Brown, M. P., Lickliter, J. D., Bampton, D., Hammond, E., Lin, L., Yang, Y., and Millward, M. (2017) An open-label, multi-center phase I study of the safety and tolerability of the novel immunomodulatory agent PG545 in subjects with advanced solid tumors. I. Clin. Oncol. 35, 3083-3084.

(27) O’Reilly, E. M., Roach, J., Miller, P., Yu, K. H., Tjan, C., Rosano, M., Krause, S., Avery, W., Wolf, J., Flaherty, K., Nix, D., and Ryan, D. P. (2017) Safety, Pharmacokinetics, Pharmacodynamics, and Antitumor Activity of Necuparanib Combined with Nab-Paclitaxel and Gemcitabine in Patients with Metastatic Pancreatic Cancer: Phase I Results. Oncologist 22, 1429-e139.

(28) Galli, M., Chatterjee, M., Grasso, M., Specchia, G., Magen, H., Einsele, H., Celeghini, I., Barbieri, P., Paoletti, D., Pace, S., Sanderson, R. D., Rambaldi, A., and Nagler, A. (2018) Phase I study of the heparanase inhibitor Roneparstat: an innovative approach for multiple myeloma therapy. Haematol. I. 103, e469-e472.

(29) Zhou, H., Roy, S., Cochran, E., Zouaoui, R., Chu, C. L., Duffner, J., Zhao, G., Smith, S., Galcheva-Gargova, Z., Karlgren, J., Dussault, N., Kwan, R. Y. Q., Moy, E., Barnes, M., Long, A., Honan, C., Qi, Y. W., Shriver, Z., Ganguly, T., Schultes, B., Venkataraman, G., and Kishimoto, T. K. (2011) M402, a Novel Heparan Sulfate Mimetic, Targets Multiple Pathways Implicated in Tumor Progression and Metastasis. PLoS One 6, No. e21106.

(30) Hammond, E., Handley, P., Dredge, K., and Bytheway, I. (2013) Mechanisms of heparanase inhibition by the heparan sulfate mimetic PG545 and three structural analogues. FEBS Open Bio 3, 346-351.

(31) Goldberg, R., Meirovitz, A., Hirshoren, N., Bulvik, R., Binder, A., Rubinstein, A. M., and Elkin, M. (2013) Versatile role of heparanase in inflammation. Matrix Biol. 32, 234-240.
(32) Gil, N., Goldberg, R., Neuman, T., Garsen, M., Zcharia, E., Rubinstein, A. M., van Kuppevelt, T., Meirovitz, A., Pisano, C., Li, J.P., van der Vlag, J., Vlodavsky, I., and Elkin, M. (2012) Heparanase Is Essential for the Development of Diabetic Nephropathy in Mice. Diabetes 61, 208-216.

(33) Secchi, M. F., Crescenzi, M., Masola, V., Russo, F. P., Floreani, A., and Onisto, M. (2017) Heparanase and macrophage interplay in the onset of liver fibrosis. Sci. Rep. 7, 14956.

(34) Thakkar, N., Yadavalli, T., Jaishankar, D., and Shukla, D. (2017) Emerging Roles of Heparanase in Viral Pathogenesis. Pathogens 6, 43.

(35) Supramaniam, A., Liu, X., Ferro, V., and Herrero, L. J. (2018) Prophylactic Antiheparanase Activity by PG545 Is Antiviral In Vitro and Protects against Ross River Virus Disease in Mice. Antimicrob. Agents Chemother. 62, e01959-17.

\section{NOTE ADDED AFTER ASAP PUBLICATION}

This paper was published on November 29, 2018. The structure of compound 29 was corrected in all parts of the paper. The revised version re-posted on December 4, 2018. 